\title{
Capacity of mosquitoes to transmit malaria depends on larval environment
}

\author{
Lillian L Moller-Jacobs ${ }^{1 *}$, Courtney C Murdock ${ }^{1,2}$ and Matthew B Thomas ${ }^{1}$
}

\begin{abstract}
Background: Adult traits of holometabolous insects such as reproduction and survival can be shaped by conditions experienced during larval development. These "carry-over" effects influence not only individual life history and fitness, but can also impact interactions between insect hosts and parasites. Despite this, the implications of larval conditions for the transmission of human, wildlife and plant diseases that are vectored by insects remain poorly understood.

Methods: We used Anopheles stephensi mosquitoes and the rodent malaria, Plasmodium yoelii yoelii, to investigate whether quality of larval habitat influenced vectorial capacity of adult mosquitoes. Larvae were reared under two dietary conditions; one group received a diet commonly used for colony maintenance $(0.3 \mathrm{mg} / \mathrm{individual} /$ day of Tetrafin fish food) while the other group received a reduced food diet $(0.1 \mathrm{mg} /$ individual/day). Upon emergence, adults were provided an infectious blood feed. We assessed the effects of diet on a range of larval and adult traits including larval development times and survival, number of emerging adults, adult body size and survival, gonotrophic cycle length, and mating success. We also estimated the effects of larval diet on parasite infection rates and growth kinetics within the adult mosquitoes.

Results: Larval dietary regime affected larval survival and development, as well as size, reproductive success and survival of adult mosquitoes. Larval diet also affected the intensity of initial Plasmodium infection (oocyst stage) and parasite replication, but without differences in overall infection prevalence at either the oocyst or sporozoite stage.

Conclusions: Together, the combined effects led to a relative reduction in vectorial capacity (a measure of the transmission potential of a mosquito population) in the low food treatment of $70 \%$. This study highlights the need to consider environmental variation at the larval stages to better understand transmission dynamics and control of vectorborne diseases.
\end{abstract}

Keywords: Anopheles stephensi, Disease ecology, Food stress, Host-parasite interactions, Nutrition, Plasmodium yoelii yoelii, Trans-stadial effects

\section{Background}

Malaria is the most important vector-borne disease of humans worldwide, with approximately 219 million people infected annually, resulting in about 600,000 deaths per year [1]. The transmission intensity of malaria is inextricably linked to the biology of the mosquito vectors and can be characterized using a summary metric known as the vectorial capacity $(C)$. Vectorial capacity describes the rate at which future infections arise from a currently infected host (provided that all female mosquitoes become

\footnotetext{
*Correspondence: IIm233@psu.edu

${ }^{1}$ Center for Infectious Disease Dynamics and Department of Entomology, Merkle Lab, Pennsylvania State University, Orchard Road, University Park, PA 16802, USA

Full list of author information is available at the end of the article
}

infected) and provides a measure of the transmission potential of a vector population $[2,3]$. It is defined as:

$$
C=\frac{m a^{2} b p^{n}}{-\ln (p)}
$$

where $m$ is vector density (ratio of adult mosquitoes to humans), $a$ is the daily probability of a human host being fed on by a vector, $n$ is the extrinsic incubation period of the parasite, $p$ is the daily probability of adult vector survival, and $b$ is the proportion of mosquitoes with sporozoites disseminated in their salivary glands.

Any variation in environment that affects relevant aspects of vector biology could result in a change in transmission risk via effects on vectorial capacity [4-8]. Recent 
work shows that changes in temperature (both means and diurnal fluctuation) and rainfall events can have substantial effects on the transmission potential of malaria [7,9-11]. Other sources of environmental heterogeneity include differences in food resource availability, seasonality of habitats, and land use changes [12,13].

To date, many studies examining the effect of environment on mosquito biology and aspects of vectorial capacity have focused directly on the adult mosquitoes. This is logical, as it is only the adult female mosquitoes that transmit malaria and the frontline interventions used for control (such as insecticide treated bed nets, indoor insecticide sprays, screening, repellents etc.) primarily target the adult stage. Small changes in daily survival probability $(p)$ and human biting rate $(a)$, for example, can have very large effects on vectorial capacity, which explains in-part the effectiveness of insecticide treated nets as these act on both traits simultaneously [14-18]. Biting rate (determined by the duration of the gonotrophic cycle) has also been shown to be a major factor explaining variation in malaria incidence [18-20]. However, larval condition has become increasingly recognized as having an influence on adult mosquito life history traits [14-16,19-21]. Based on studies in other invertebrate systems, it is expected that variation in quality of larval habitats could feed through to impact adult life history, which in turn could affect transmission [22-24]. For terrestrial insects and other small invertebrates, estimates of body condition are often positively correlated with body size [25,26], and larger individuals often exhibit increased probability of survival, fecundity and ultimate overall fitness $[27,28]$. Adult survival and vector density are key elements of vectorial capacity. Larval effects on adult body size might also be important in determining vector competence. Larger individuals could support more parasites due to greater availability of host resources $[29,30]$. Alternatively, individuals with more reserves might be able to devote more energy toward immune defense [19,31].

Extensive studies on Aedes species have shown that larval environment can have considerable effects on life history traits important to transmission, such as development rate, adult longevity, and efficiency of egg development [32-34]. Larval environment has also been demonstrated to significantly shape vector competence in a variety of Aedes-arbovirus systems. For example, interactions between larval competition and density in Aedes aegypti and Aedes albopictus can significantly increase susceptibility to dengue virus and Sindbis virus $[35,36]$. Nutritional stress in Aedes aegypti has also been shown to influence the interaction between humoral and cellular branches of the immune system, which could affect vector competence for a suite of pathogens [37].

A more limited number of studies on Anopheles vector spp. support the influence of larval condition on subsequent adult traits and vectorial capacity, but the patterns are not well understood [15,38-40]. Here, we use the Asian malaria vector, Anopheles stephensi (Liston), and a rodent model malaria, Plasmodium yoelii yoelii, to investigate whether nutritional quality of larval habitat affects vectorial capacity. We show trans-stadial impacts on a range of traits indicating potential for strong effects of larval rearing condition on subsequent transmission of malaria.

\section{Methods}

\section{General experimental design}

To manipulate larval environment quality, we fed $A n$. stephensi larvae differing quantities of food throughout development. Larvae were collected from our standard lab colony at The Pennsylvania State University (this colony was initiated in 2011 with eggs from a longstanding colony maintained at Johns Hopkins University). Newly hatched $(<24 \mathrm{~h}$ old $)$ first instar larvae were transferred to $265 \mathrm{~mL}$ plastic cups containing $80 \mathrm{~mL}$ of distilled water at initial densities of 50 larvae per cup. Larvae were maintained on Tetrafin ${ }^{\circledR}$ fish food under standard insectary conditions $\left(26^{\circ} \mathrm{C} \pm 0.5^{\circ} \mathrm{C}, 80 \%\right.$ humidity, and a 12:12 L:D photoperiod). We exposed larvae to one of two experimental food treatments: 1) a "high" food regime $(0.3 \mathrm{mg} /$ individual/day), which is consistent with our standard colony maintenance, and 2) a "low" food regime $(0.1 \mathrm{mg} /$ individual/day). These food treatments were selected based on a series of pilot studies. We maintained a constant food environment in each cup by counting larvae and pupae daily, replacing water (kept at a constant volume), and adjusting the amount of food allocated per individual. Each treatment group contained 48 replicate cups. The entire experiment was repeated in time, for a total of two experimental blocks (96 cups per treatment, in all). For mating success and gonotrophic cycle experiments (details below), each larval treatment group contained 15 cups in two experimental blocks (also repeated in time), totaling 30 cups per treatment.

\section{Quantifying the effects of food treatment on mosquito life history traits \\ Larval survival and development time}

To assess effects of experimental food treatment on larval survival, larvae and pupae in each cup were counted daily using an eyedropper. To estimate duration of larval development, and quantify adult mosquito production of each cup, the date of adult female emergence and number of females emerging were recorded.

\section{Adult body size}

To determine if larval food manipulation generated differences in adult female body size, we took one wing per mosquito ( $\mathrm{n}=100$ per treatment, per experimental block) 
and mounted wings onto a glass microscope slide using clear nail lacquer. Wings were then measured using a micrometer eyepiece with a standard dissecting microscope. Measurements were taken from the tip of the wing (excluding fringe) to the distal end of the alula. Wing length is known to be positively correlated with body size in mosquitoes $[41,42]$.

\section{Gonotrophic cycle, mating success and daily adult survival}

To determine effect of larval food treatment on gonotrophic cycle length, females that had emerged 3-5 days prior (and had been housed in mesh cages with males from their respective treatments) were fed to repletion on a Hemotek membrane feeder using pork sausage casing filled with human blood heated to $37^{\circ} \mathrm{C}$. Blood-fed females $(n=50$ per treatment in each experimental block, for a total of 100 per treatment) were then transferred to individual $50 \mathrm{~mL}$ plastic tubes covered with mesh, and provided with a cotton ball moistened with $10 \%$ glucose solution that was replenished daily. Each day, sugar moistened cotton balls were removed 4 hours prior to offering a blood meal (also via a Hemotek feeder) for 10 minutes each on four subsequent days. Tubes were monitored daily for eggs, which were counted for each individual female. Mortality was recorded until day 14 after the first blood meal.

Here, gonotrophic cycle is defined as the time (in days) from initial blood meal to laying of the first clutch of eggs, as few individuals laid twice throughout the experiment. Mating success was also assayed by dissection of spermathecae from females in both treatments that never laid eggs throughout the monitoring period. Presence of sperm, whether alive or dead, was considered a successful mating.

Adult survival was monitored for 14 days after the first blood meal, at which point adult females were 17-19 days old. While mosquitoes can live for many weeks under ideal lab conditions [10], this prolonged survival is difficult to interpret as it is generally accepted that few mosquitoes live beyond 2 weeks in field settings [43-45]. Moreover, 14 days after the blood meal is when female mosquitoes are potentially able to transmit malaria and before strong effects of senescence are expected (note the basic vectorial capacity equation is age-independent and assumes a constant rate of mortality in the absence of senescence).

\section{Quantifying the effects of food treatment on vector competence}

In order to assess the effects of larval food treatment on measures of vector competence, we first randomly allocated three-to-five day old individuals from each cup of both treatments across one of four replicate cages (80-100 females per cage). Females were allowed to feed on two anesthetized mice per cage (C57 females, > 6 weeks old) infected with Plasmodium yoelii yoelii (clone 17XNL) for thirty minutes. All vertebrate animal work was carried out by trained research technologists under Penn State University IACUC protocols specified in permit \#27452. To ensure that mosquitoes received an infectious blood meal at the same adult age, first instar larvae for the low food treatment group were collected from the colony four days earlier than first instars to be allocated to the optimal food treatment group. This was done to adjust for the slower developmental time in the low food treatment group and allow for age-matched comparison of the groups at the same time after administration of the infectious blood meal. Individuals that did not feed were removed from the cage. Infectious feeds were performed at $26^{\circ} \mathrm{C}$ for optimum host seeking and probing behavior. Immediately following feeding, cages were transferred to a second incubator set at $24^{\circ} \mathrm{C}$, as this is the thermal optimum for Plasmodium yoelii yoelii growth and replication. After infection, individuals were provided with cotton balls moistened with $10 \%$ glucose offered ad libitum and replaced daily. Daily survival of infected adults was monitored by counting and removing the dead individuals throughout the duration of the experiment.

Despite our high level of replication at the larval food level (48 cups per treatment per block), we chose to pool individuals from cups into cages (4 per treatment per block). Combining in this way means we cannot trace individual mosquitoes and their resulting infection dynamics back to a specific cup. However, we considered randomization across 4 treatment cages, with use of 2 mice per cage to account for natural mouse-to-mouse variation in gametocytemia, and complete replication through time, sufficient to detect any biologically relevant treatment effects. Putting individual mice on each cup would have increased the number of mice used to unethical levels.

\section{Parasite prevalence and intensity}

A subset of 25 individuals was taken from each replicate cage at seven days post-infection to assess infection prevalence and intensity. Midguts were dissected in $1 \mathrm{X}$ phosphate-buffered saline and examined under a light microscope. Presence or absence of oocysts and the number of established oocysts were quantified. Dissected midguts were saved individually in $1.5 \mathrm{~mL}$ tubes and stored in absolute ethanol at $-80^{\circ} \mathrm{C}$ for future genomic DNA analysis (discussed below). At day 16-18 postinfection, 15 individuals were sampled from each replicate cage, and salivary glands were dissected out in $1 \mathrm{X}$ phosphate-buffered saline. Glands were examined under a light microscope and scored for presence or absence of sporozoites. To estimate vector competence ( $b$, proportion of infectious bites on a susceptible host that lead to 
an infected host), we used the proportion of mosquitoes with sporozoites disseminated in the salivary glands. This is a standard approximation and assumes that if a mosquito has sporozoites in the salivary glands, it will likely transmit during feeding $[3,4,46]$.

\section{Sporozoite replication}

To quantify how food treatment affected sporozoite production for each infected mosquito, we performed genomic DNA extraction and qPCR analysis for Plasmodium genomes in midguts saved from oocyst dissection. Plasmodium genomic DNA was extracted from midguts using the E.Z.N.A. MicroElute Genomic DNA kit (Omega BioTek, as per the manufacturer's protocol). DNA was eluted in $20 \mu \mathrm{L}$ of molecular grade water, and the number of parasite genomes present in midguts was quantified using a previously developed qPCR assay [47]. Briefly, reactions were run on an ABI Prism 7500 Sequence Detection System (TaqMan). Initial denaturation was 20 seconds at $95^{\circ} \mathrm{C}$ followed by 40 cycles of a three-second $95^{\circ} \mathrm{C}$ denaturation period and a 30 -second $60^{\circ} \mathrm{C}$ period of annealing and extension. Primers and probes were designed to amplify DNA from several Plasmodium species. We constructed standard curves for $P$. yoelii genome detection by extracting DNA from a known number of infected mouse red blood cells using the BloodPrep kit (Applied Biosystems) on the ABI Prism 6100 Nucleic Acid Prep Station (as per the manufacturer's protocol). Parasite production per oocyst was evaluated by dividing the total number of parasite genomes by the number of oocysts quantified for each midgut. We used both sporozoite production per midgut and per oocyst as measures of the efficiency of parasite replication.

\section{Statistical analyses}

All statistical analyses were carried out using IBM SPSS v.21 (Armonk, NY). For all analyses, full factorial models were reduced through backwards elimination of nonsignificant, higher order interactions, and henceforth nonsignificant higher order interactions are not reported in our discussion of the results or displayed in our model tables. All models were evaluated for goodness of fit by assessing model deviance per degrees of freedom, log likelihood values and residual plots. To assess significant pairwise comparisons, we used Bonferroni-adjusted post-hoc tests.

We used univariate general linear model (GLM) analysis to determine how food treatment affected adult body size and mating success. Larval and adult survival, day of emergence, number of adults emerged, fecundity and measures of vector competence (oocyst intensity and prevalence, sporozoite prevalence) were analyzed using generalized linear models (GZLM) so that nonnormal error distributions could be used in the analysis. All distributions were chosen based on both best model fit and plots of raw data.

Normal distribution was assumed for analysis of day of adult female emergence and gonotrophic cycle. We assumed complimentary log-log, poisson, binomial, negative binomial, and gamma distributions in the analyses for mosquito survival, number of adults emerging and fecundity, oocyst and sporozoite prevalence, oocyst intensity, and number of sporozoites produced for each treatment group, respectively. For each dependent variable in our analyses, food treatment, cage replicate (for parasite traits), and experimental block were included as factors. We included oocyst intensity as a covariate in the model assessing treatment effects on sporozoite production. Across all models concerning characteristics of Plasmodium infection, replicate cage was nested within treatment to correct for the fact that mosquitoes in each cage received a blood meal from a different group of mice, and so were not related to one another across treatment groups.

\section{Quantifying effects of larval food treatment in the context of the vectorial capacity equation}

We calculated vectorial capacity using mean parameter estimates quantified from our empirical data (Table 1). In the current study we have no direct measure of the extrinsic incubation period (EIP) of the parasite $(n)$, so we assumed the EIP for $P$. yoelii development at $24^{\circ} \mathrm{C}$ to be 12 days for both treatment groups based on previous research [4]. For daily survival rates $(p)$, we used the average rate over the entire 18-day monitoring period. Vector density $(m)$ was estimated using the mean of total emerged females per replicate larval cup. We followed convention in using the reciprocal of the mean gonotrophic cycle length as a proxy for daily biting rate (a). The proportion of mosquitoes potentially infectious (b), was calculated using raw data means for sporozoite prevalence.

All values were calculated using means from our empirical data, except for extrinsic incubation period (EIP), which is assumed based on ideal conditions in previous work. Standard errors of means are displayed for vector

Table 1 Output of vectorial capacity equation with experimental parameters

\begin{tabular}{|c|c|c|c|c|c|c|}
\hline Treatment & Adult vector density $(m)$ & Biting rate $(a)$ & Adult daily survival $(p)$ & Proportion infectious $(b)$ & $\operatorname{EIP}(n)$ & Vectorial capacity $(C)$ \\
\hline Low diet & $16.7 \pm 0.440$ & $0.233 \pm 0.009$ & 0.973 & $0.3 \pm 0.042$ & 12 & 7.155 \\
\hline High diet & $19.7 \pm 0.359$ & $0.293 \pm 0.006$ & 0.982 & $0.3 \pm 0.042$ & 12 & 22.462 \\
\hline
\end{tabular}



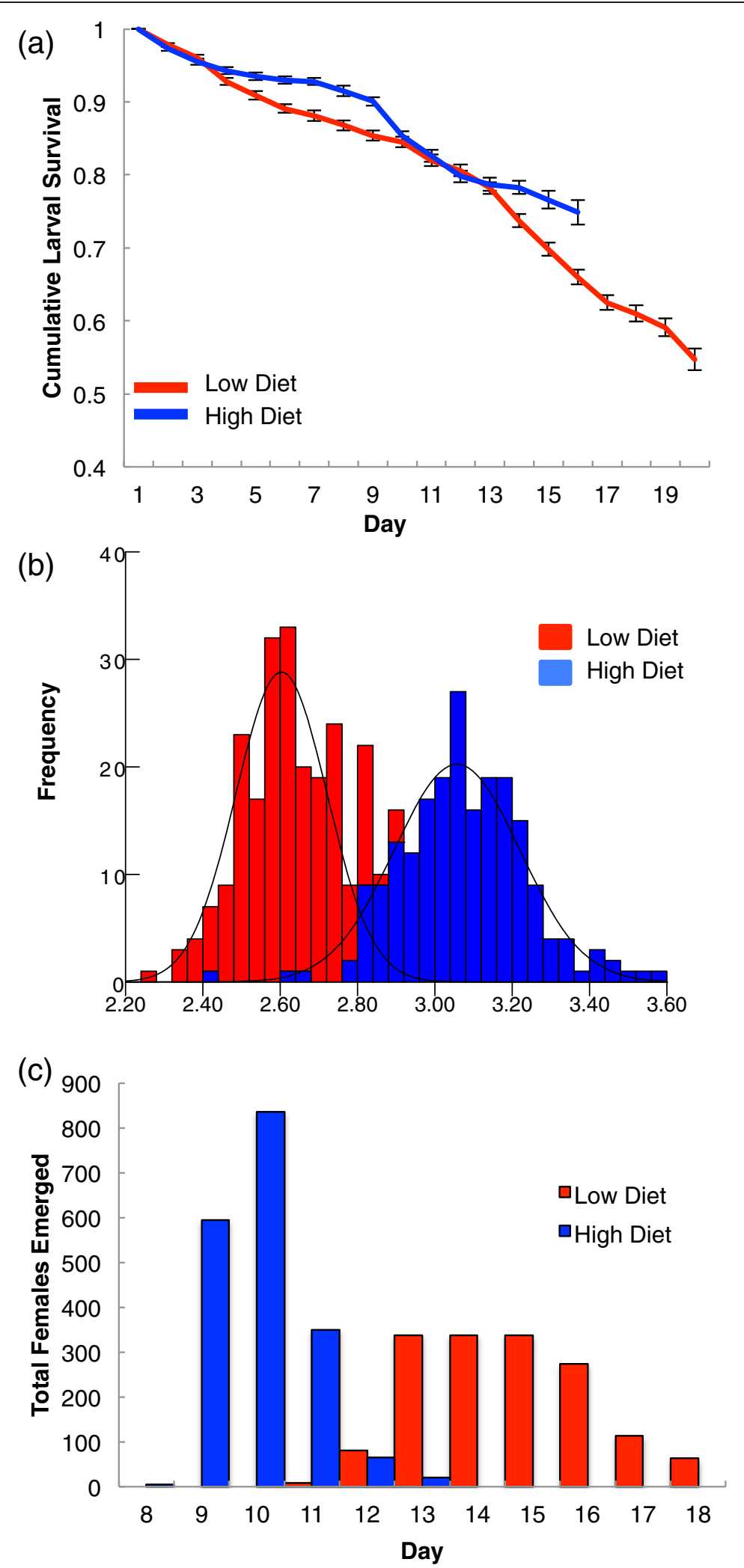

Figure 1 (See legend on next page.) 
(See figure on previous page.)

Figure 1 Effects of larval nutrition on larval development time, adult body size, and day of female emergence. a. Daily survival from first instar larva to adult. End of survival curve signifies all adults have emerged $\mathbf{b}$. Frequency distribution of wing length in females across both treatment groups (red =0.1 mg/individual/day, $n=213$, blue $=0.3 \mathrm{mg} /$ individual/day, $n=206$ ). Groups are significantly different from each other (treatment, $p=<0.001$, univariate GLM). b. Daily survival from first instar larva to adult. End of survival curve signifies all adults have emerged. c. Female emergence per day; depicts total adults (all replicates, all blocks) emerged at each day after hatching. Female emergence in the low diet is characterized by fewer individuals, as well as a more extended pattern of emergence with no distinct peak.

density, biting rate, and proportion infectious (sporozoite prevalence).

\section{Results}

\section{The effects of food treatment on mosquito life history} traits

Food treatment significantly affected larval survival (and henceforth the number of emerging adult females). Larvae reared on low food diets did not survive as well as those reared on high food diets (Figure 1a). However, this effect was strongly mediated by day (analyzed here as a covariate) and interactions among food treatment and experimental block and experimental block and day (Table 2, GZLM, interval-censored survival analysis). Although the trend of reduced larval survival and subsequent adult emergence in the low food group remained consistent across experimental blocks, the first block showed an initial (days 2-5 after hatching) sharp decline in the survival of the high food group, which did not replicate in the second experimental block resulting in the significant treatment $x$ day $x$ block interaction (Table 2). We did see significant replicate effects; however, upon plotting Kaplan-Meier survival curves for each of the 48 replicates per treatment per block, these effects were due to several cups that exhibited unusually high or low larval mortality (data not shown). However, the trends remain the same and when assessing the final productivity of each cup (total number of females), replicate is not a significant effect.
Low food environments produced, on average, significantly fewer total adult females per replicate cup than the high food environments (Figure 1c). In the low diet, mean emergence was 16.7 females (S.E. $=0.44)$, while high diet cups produced a mean of $19.7($ S.E. $=0.359)(\mathrm{p}=<0.001$, GZLM, Table 2). Adult females emerging from the high food treatment group had significantly larger wings than those emerging from the low food treatment group, with little overlap between the two groups (Figure 1b).

Length of gonotrophic cycle was significantly shaped by larval food treatment (Table 2), and females from low larval food diets had gonotrophic cycles that were on average one day longer than mosquitoes fed high food diets (Figure 2a). There was a significant effect of experimental block, with individuals in the second taking slightly longer to lay the first clutch of eggs, but this effect was consistent across treatments, resulting in no significant treatment $x$ block interactions. Larval food treatment also significantly affected mating success (presence of sperm in spermatheca; Figure 2b) and fecundity. Females from the low food treatment were less likely to be mated than those from the high food treatment (mean proportion mated $=0.71 \pm .45,0.97 \pm 0.18$, respectively), and those in the low food treatment that did lay eggs, laid significantly fewer than high food females (mean size of first clutch $=28.67 \pm 2.011,64.14 \pm$ 1.797, respectively; treatment $\mathrm{p}<0.001$, GZLM, Poisson distribution).

Table 2 Generalized linear model output for mosquito life history traits

\begin{tabular}{|c|c|c|c|c|c|c|c|c|c|c|c|c|}
\hline \multirow[b]{2}{*}{ Factors } & \multicolumn{3}{|c|}{ Larval survival $(\mathrm{n}=3177)$} & \multicolumn{3}{|c|}{ Vector density $(n=190)$} & \multicolumn{3}{|c|}{$\begin{array}{l}\text { Adult survival larval survival } \\
(\mathrm{n}=414)\end{array}$} & \multicolumn{3}{|c|}{ Gonotrophic cycle $(n=145)$} \\
\hline & Wald $X 2$ & d.f. & $p$ - value & Wald $X 2$ & d.f. & $p$ - value & Wald $X 2$ & d.f. & $p$ - value & Wald $X 2$ & d.f. & $p$ - value \\
\hline Intercept & $a$ & $a$ & 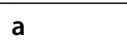 & 7817.07 & 1 & $<0.001$ & 122.43 & 1 & $<0.001$ & 2713.148 & 1 & $<0.001$ \\
\hline Treatment & 78.06 & 1 & $<0.001$ & 114.46 & 1 & $<0.001$ & 11.63 & 1 & $<0.001$ & 35.687 & 1 & $<0.001$ \\
\hline Replicate (treatment) & 768.72 & 94 & $<0.001$ & 99.03 & 94 & $<0.001$ & 4.36 & 6 & 0.629 & N.A. & N.A. & N.A. \\
\hline Block & 61.12 & 1 & $<0.001$ & 32.69 & 1 & 0.341 & 18.34 & 1 & $<0.001$ & 9.895 & 1 & 0.002 \\
\hline Day & 1815.62 & 1 & $<0.001$ & N.A & N.A & N.A & 355.22 & 1 & $<0.001$ & N.A. & N.A. & N.A. \\
\hline Treatment $x$ block & 50.545 & 1 & $<0.001$ & N.S & N.S & N.S & N.S & N.S & N.S & N.S. & N.S. & N.S. \\
\hline Treatment $x$ day & 0.334 & 1 & 0.563 & N.A & N.A & N.A & N.S & N.S & N.S & N.A. & N.A. & N.A. \\
\hline Block $x$ day & 27.485 & 1 & $<0.001$ & N.A & N.A & N.A & N.S & N.S & N.S & N.A. & N.A. & N.A. \\
\hline Treatment $x$ block $x$ day & 32.537 & 1 & $<0.001$ & N.S & N.S & N.S & N.S & N.S & N.S & N.A. & N.A.1 & N.A. \\
\hline
\end{tabular}

Bold indicates significance at $a=0.05$. (Model fit assessed by value of deviance per degrees of freedom: larval survival $=1.450$, vector density $=0.934$, adult survival $=8.967$, gonotrophic cycle $=0.765$ ). P-values are reported only for significant interactions and first order terms. Table reflects the final output of a backwards-eliminated full factorial model. N.S. = not significtant, N.A. = not applicable, $a=$ SPSS unable to compute due to numerical issues. 


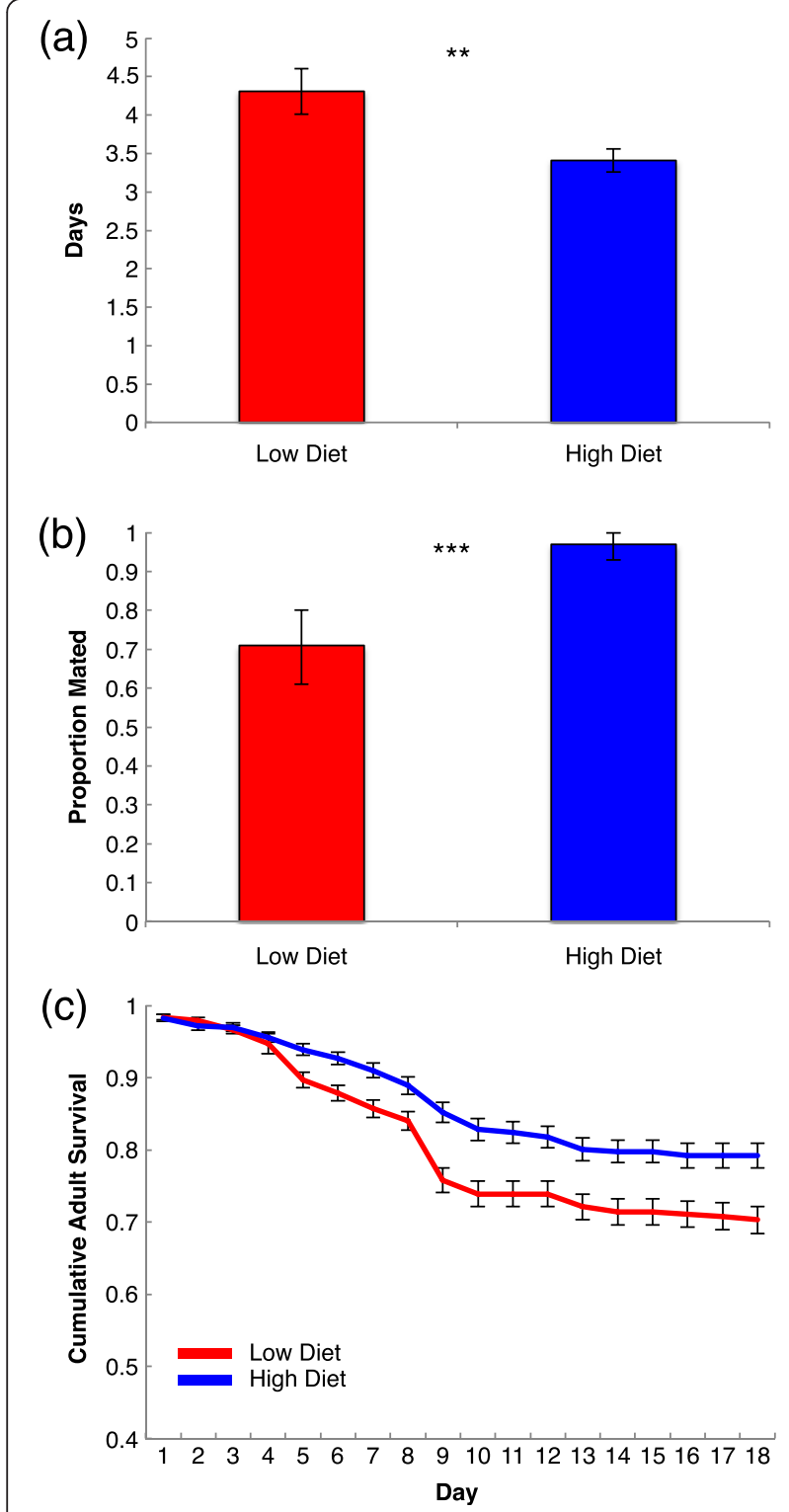

Figure 2 Effects of larval nutrition on adult female reproductive traits and post-infectious survival. a. Length of first gonotrophic cycle, used to estimate biting rate. Treatments are significantly different from one another ( $p=<0.001$, univariate $G L M)$. Bars represent $95 \%$ confidence intervals around mean values. Low diet $n=55$, high diet $n=90$. $\mathbf{b}$. Mean proportion of individuals with sperm present in spermathecae. Individuals from optimal food treatments exhibited higher mating success than those from low food treatments. Bars are significantly different from one another $(p=<0.001)$. Error bars represent the $95 \%$ confidence intervals around the mean values. Low diet $n=85$, high diet $n=93$. c. Daily post infectious adult female survival of each group. Note the most significant changes occur between day four and ten.

\section{Effects of food treatment on vector competence}

Larval food treatment significantly affected adult infected female survival ( $\mathrm{p}=0.001$, GZLM, interval-censored survival, Table 2). The difference was not significant until day five post-infection, when survival in the low food group fell sharply, then leveled off at day 10, with little mortality in either group for the remainder of the observation period (Figure 1c).

Despite differences in adult survivorship, food treatment had no significant effect on either oocyst or sporozoite prevalence (Table 3, Figure 3a). In contrast, food treatment significantly influenced oocyst intensity (Table 3, Figure $3 \mathrm{~b}$ ), and females from the low food group had an overall lower oocyst burden than those from the high larval food group. This effect was consistent across experimental blocks, despite the difference in infection levels between experiments.

Individuals fed a high food diet exhibited a greater number of parasite genomes per oocyst than mosquitoes fed a low food diet (mean of $1.69 \times 10^{5}$ and $2.2 \times 10^{5}$ genomes per midgut for low and high treatment, respectively) (Table 3). Unsurprisingly, the number of Plasmodium genomes per midgut increased with oocyst intensity. However, the slope of the positive relationship between oocyst intensity and number of Plasmodium genomes is steeper for individuals in the high food treatment (slope $=9.38 \mathrm{E} 3$, $\mathrm{R}^{2}=0.787$ ) than for the low food treatment (slope $=$ $6.44 \mathrm{E} 3, \mathrm{R}^{2}=0.747$ ), as indicated by the significant interaction between food treatment and oocyst intensity (treatment $x$ oocyst intensity, Table 3 ).

Here, the significance of experimental block is likely due to the fact that oocyst intensity in the second block (mean = 13.26) was lower than that of the first block (mean = 46.36). Though the trends replicate and remain significant, the magnitude of the relationship between intensity and genome count is much greater in the first block.

\section{Effects of food treatment on vectorial capacity}

For daily survival rates $(p)$, we took the average rate over the entire 18-day monitoring period, which resulted in $p=$ 0.973 and $p=0.983$ for the low and high food groups, respectively. Mean adult female density $(m)$ per replicate cup in the low diet was 16.7 females (S.E. = 0.44), while high diet cups produced a mean of 19.7 (S.E. $=0.359$ ) ( $\mathrm{p}=<0.001$, GZLM, Table 2). Gonotrophic cycle for low food individuals was 4.29 days (the reciprocal of which gives an average biting rate per day of $a=0.233$, S.E. $=.009$ ), while high food individuals had an average cycle length of 3.41 days $(a=0.293$, S.E. $=0.006)$. Sporozoite prevalence $(b)$ mean was 0.30 for both groups $($ S.E. $=0.042)$. These parameter values yielded mean vectorial capacities of $C=7.155$ for mosquitoes from the low food environment and $C=22.462$ for those from the high food environment.

\section{Discussion}

In this study we demonstrate effects of variation in food abundance at the larval stage on both larval survival and a suite of adult mosquito life history traits that combine to 
Table 3 Generalized linear model output for vector competence and parasite dynamics

\begin{tabular}{|c|c|c|c|c|c|c|c|c|c|c|c|c|}
\hline \multirow[b]{2}{*}{ Factors } & \multicolumn{3}{|c|}{$\begin{array}{l}\text { Oocyst prevalence } \\
(n=417)\end{array}$} & \multicolumn{3}{|c|}{$\begin{array}{l}\text { Sporozoite prevalence } \\
(\mathrm{n}=239)\end{array}$} & \multicolumn{3}{|c|}{$\begin{array}{l}\text { Oocyst intensity } \\
(\mathrm{n}=316)\end{array}$} & \multicolumn{3}{|c|}{$\begin{array}{l}\text { Plasmodium genomes per midgut } \\
(\mathrm{n}=316)\end{array}$} \\
\hline & Wald $X 2$ & d.f. & $p$ - value & Wald $X 2$ & d.f. & $p$ - value & Wald $X 2$ & d.f. & $p$ - value & Wald $X 2$ & d.f. & $p$ - value \\
\hline Intercept & 104.07 & 1 & $<0.001$ & 35.91 & 1 & $<0.001$ & 2535.06 & 1 & $<0.001$ & 14425.51 & 1 & $<0.001$ \\
\hline Treatment & 1.93 & 1 & 0.164 & 0.01 & 1 & 0.944 & 76.61 & 1 & $<0.001$ & 43.42 & 1 & $<0.001$ \\
\hline Replicate(treatment) & 5.57 & 6 & 0.473 & 9.04 & 6 & 0.171 & 20.79 & 6 & 0.002 & 2.20 & 6 & 0.900 \\
\hline Block & 35.91 & 1 & $<0.001$ & 12.20 & 1 & $<0.001$ & 79.3 & 1 & $<0.001$ & 74.68 & 1 & $<0.001$ \\
\hline Intensity & N.A. & N.A. & N.A. & N.A. & N.A. & N.A. & N.A. & N.A. & N.A. & 114.83 & 1 & $<0.001$ \\
\hline Treatment $x$ block & N.S. & N.S. & N.S. & N.S. & N.S. & N.S. & 33.79 & 7 & $<0.001$ & 7.18 & 1 & $<0.001$ \\
\hline Treatment $x$ intensity & N.A. & N.A. & N.A. & N.A. & N.A. & N.A. & N.A. & N.A. & N.A. & 16.15 & 1 & $<0.001$ \\
\hline Block $x$ intensity & N.A. & N.A. & N.A. & N.A. & N.A. & N.A. & N.A. & N.A. & N.A. & 35.96 & 1 & $<0.001$ \\
\hline
\end{tabular}

Bold indicates significance at $a=0.05$. (Model fit assessed by value of deviance per degrees of freedom: oocyst prevalence $=1.379$, sporozoite prevalence $=1.748$, oocyst intensity $=1.422$, genomes per midgut $=1.286$ ) .

P-values are reported only for significant interactions and first order terms. Table reflects the final output of a backwards-eliminated full factorial model. Intensity is included as a covariate only for Plasmodium genomes, as it would be an inappropriate variable for all other parasite traits reported in this table. N.S. = not significtant, N.A. $=$ not applicable.

determine vectorial capacity. The treatment effects equate to a $c .70 \%$ relative reduction in malaria transmission potential for mosquitoes from the low food environment, or conversely, a relative increase in transmission potential of c.310\% for mosquitoes from the high food environment.

The most obvious influence of larval food treatment was the impact on daily survival and prolonged development of larvae in poor nutritive environments. Longer development times and smaller proportion of mosquitoes successfully pupating and eclosing, has been reported in Anopheles gambiae, Aedes triseriatus and Anopheles darlingi $[14,48,49]$. Further, although larval development is not a direct component of vectorial capacity, it will affect vector population growth rates and possibly vector density (depending on the nature of the density dependence [50]). Adult survival has also been shown to be affected by quality of larval habitat in other mosquitoes [51,52]. One recent study suggested that quality of larval habitat had no effect on subsequent survival of adult An. stephensi and hence, no implications for transmission [39]. However, this study monitored adult survival for 5 days only, which is much shorter than the incubation period of the parasite and so provides little insight into ultimate impacts on the number of adults potentially able to transmit the parasite (note that differences in survival between treatments in our study only emerged between days 5-9).

Larval food treatment also carried over to have a significant effect on adult body size. Several studies have shown positive correlation between body size and fitness in vector species. For example, in Aedes albopictus reared in both the laboratory and the field, pupal mass and wing length were consistently correlated positively with fecundity [53]. In field-collected Aedes communis, wing length was a significant predictor of longevity when the adult females were food stressed [54]. Studies on
Anopheles gambiae show that reduction in resource availability through increasing larval density significantly decreases adult mass in males and females. Reduced body mass was demonstrated to be negatively correlated with both age at pupation and mating success, suggesting that both development time and body size have an influence on adult fitness [55-57].

Larval food treatment also impacted duration of the gonotophic cycle, and hence the reciprocal estimate of daily biting rate. Changes in gonotrophic cycle length were relatively small ( $<1$ day) but because biting rate enters into the vectorial capacity equation as a squared term (reflecting that one bite is required for a mosquito to acquire the parasite and at least one other required to pass it on), even small changes can contribute to differences in overall vectorial capacity $[18,58,59]$. The prolonged gonotrophic cycle length we observe in small females could be due to decreased nutritional reserves (as could the effects on fecundity). This reduction in reserves has been shown in Aedes aegypti females to prolong especially the first gonotrophic cycle, as more than $50 \%$ of the lipid resources needed to develop eggs are from larval stores [33]. In nature, the timing of the initial blood meal could also be delayed if a smaller proportion of females engage in blood feeding, as seen in Anopheles darlingi emerging from poor larval habitats [40], or if smaller females have higher initial preference for sugar feeding as opposed to blood feeding [60]. These latter effects would potentially further impact vectorial capacity.

Differences in fecundity and mating success, while they do not contribute directly to vectorial capacity, could further shape vector density. It has been demonstrated in several studies that fecundity is positively correlated with body size in several anopheline species, due to both available teneral reserves for ovarian development as well as larger blood meal size taken by larger females [61-63]. 

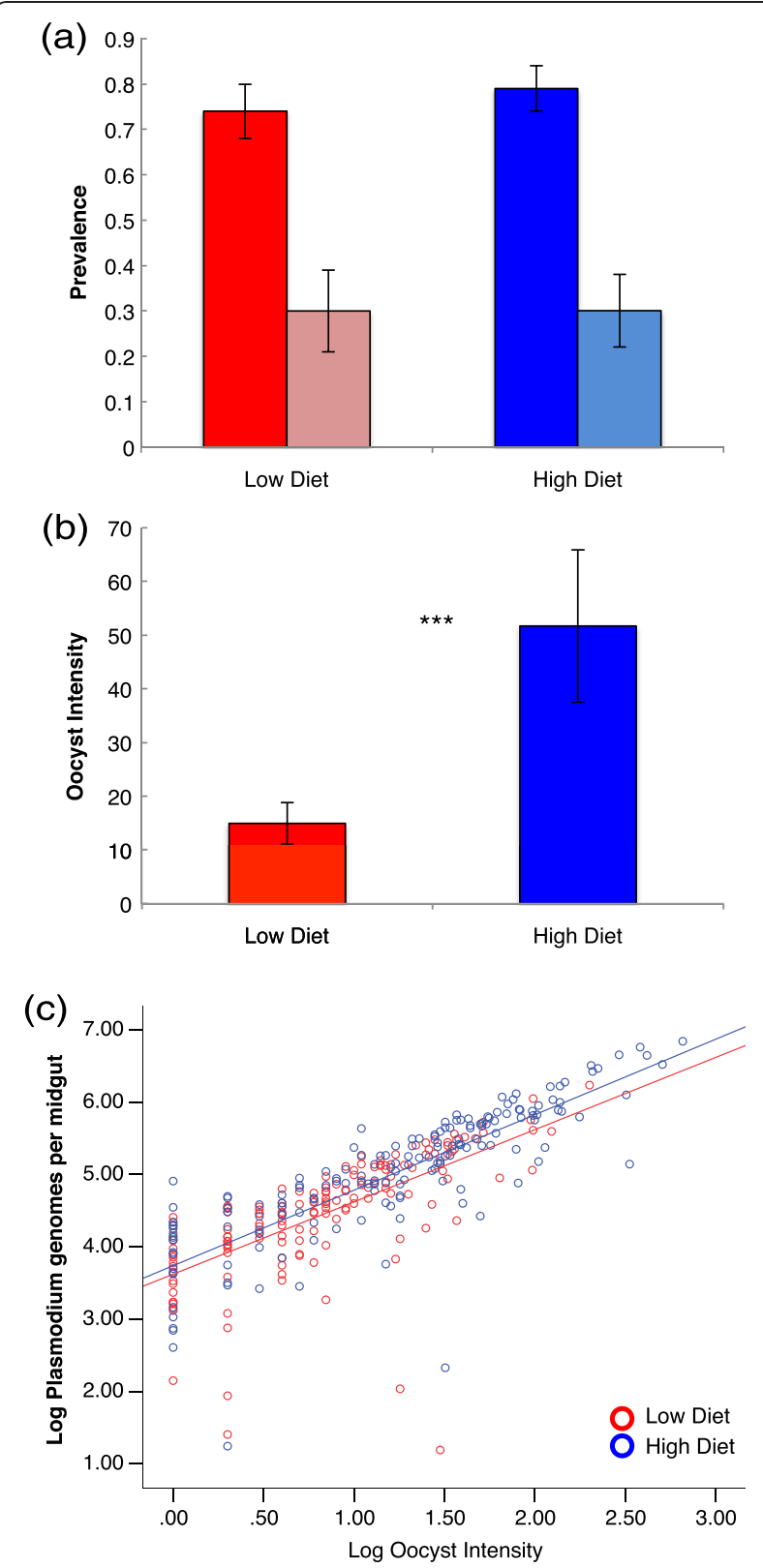

Figure 3 Effects of larval nutrition on parasite dynamics in midgut (oocyst) and salivary gland (sporozoite) stages. a.

Prevalence of oocysts (dark-colored bars) and sporozoites (light-colored bars) in both treatments. There is no significant difference between low and high food treatment individuals for oocysts ( $p=0.164$, GZLM) or sporozoites ( $p=0.944, G Z L M$ ). Error bars represent the $95 \%$ confidence intervals for mean values. $\mathbf{b}$. Infection load for each treatment as quantified by oocyst intensity in infected individuals. Treatments are significantly different from one another $(p<0.001$, GZLM). Error bars represent the 95\% confidence intervals for mean values. c. Relationship between oocyst intensity and sporozoite production (Plasmodium genomes per midgut). Both oocyst intensity and total genomes are log-transformed for clarity in visualization. Treatments are significantly different from one another ( $p<0.001, G Z L M)$.
Possible explanations for impacts on mating success include depressed mate-seeking in small individuals of both sexes due to energetic demand $[64,65]$. Anopheles gambiae males have been demonstrated to preferentially select larger females for mates [66], so low food females may have not presented as attractive mates for their conspecific males. Additionally, differences between treatments could affect the viability and number of sperm [67]. In our studies, mosquitoes were restricted to mating within their respective treatment groups. These trends in mating success might change if mosquitoes were allowed to mate freely with individuals of differing sizes from diverse larval habitats [68].

The effects of larval diet on parasite development and overall vector competence were mixed. We observed differences in parasite intensity and genomic DNA replication, suggesting that larger mosquitoes might offer greater nutritional resources for parasite exploitation within the insect, but found no effect on parasite prevalence at either the oocyst or sporozoite stage. We used a rodent malaria as an established model [69] but there are recognized differences between typical infection intensities of rodent and human malarias [70], and the resultant mosquito immune responses [71,72]. We also acknowledge that we are using a laboratory strain of $A n$. stephensi that has known susceptibility to certain Plasmodium species and that natural sympatric vectorparasite pairings can differ in baseline competence [73]. The functional importance of these differences is slightly unclear, but they complicate translation of results across systems and we do not suggest that our calculations of vectorial capacity can be applied directly to the field. That said, human malaria parasites do require mosquito resources for development [74-77] so there is no reason to think that host condition is unimportant. Several previous studies have demonstrated effects of larval density on adult traits including body size [15,55,56], survival [52], blood intake, and mating competitiveness [78,79] suggesting our results to be robust. Comparative differences might be enhanced or diminished in the face of the complexity that exists in the field.

The effects we observe on parasite replication rate suggest the additional intriguing possibility that host condition could affect the extrinsic incubation period (EIP) of the parasite. We did not measure EIP in our study as we dissected salivary glands at a single time point during infection. However, differences in parasite replication rate due to the influence of temperature, for example, are known to affect length of the EIP, with important consequences for vectorial capacity $[4,10]$ We are not aware of any studies exploring the effects of mosquito condition (larval through to adult) on malaria parasite development but nutritionally-dependent parasite replication has been observed in other invertebrate-parasite 
systems [30,80-82]. If EIP is affected by mosquito condition, this could add to the influence of larval habitat on malaria transmission.

Russell et al. (47) analysed a time series of An. gambiae abundance and condition (body size) data collected in a high transmission setting in central Tanzania. Their study revealed marked variation in adult body size across the year linked to rainfall-driven changes in availability (and likely quality) of larval breeding habitats. The density dependent feedbacks between mosquito condition and carrying capacity of larval habitats provided an elegant explanation for the observed adult mosquito population dynamics. Our data indicate that variation in quality of larval habitats could have important impacts on vectorial capacity beyond predicted effects on mosquito abundance.

\section{Conclusions}

Caveats regarding the laboratory nature of our experimental system notwithstanding, the magnitude of the effects we observe are non-trivial. A 70\% reduction in vectorial capacity is of the same order as reported from well implemented control programs using insecticide sprays or insecticide treated nets [83-86]. Thus, variation in larval habitat quality could have a marked influence on the temporal and spatial dynamics of malaria transmission. Larval resource effects could also have important implications for control. For example, poorly implemented larval control, such as inefficient larviciding, could possibly alleviate density effects, increasing resource availability for the remaining larvae. This reduced larval competition might lead to inadvertent increases in transmission potential. Conversely, reduced nutrition via increases in larval competition could reduce transmission potential and might provide a partial explanation for the so-called 'paddy paradox', whereby malaria burden remains low in rice irrigation areas in spite of very high mosquito densities [87]. The potential for such effects reinforces the need for better understanding the effects of environmental quality on larval and adult mosquito ecology [88].

\section{Competing interests}

The authors declare that they have no competing interests.

\section{Authors' contributions}

Conceived and designed the experiments: LLM-J CCM MBT. Performed the experiments: LLM-J. Analyzed the data: LLM-J CCM. Wrote the manuscript: LLM-J CCM MBT. All authors read and approved the final submitted version of this manuscript.

\section{Acknowledgments}

We thank members of the Thomas and Read laboratory groups for discussion, Andrew Read for statistical analysis advice, J. Teeple for insectary support, and M. Jones, S. Blanford and D. Sim for laboratory assistance. This research was funded by NIH-R21 (Al096036-01) and NSF-GRFP \#DGE1255832. This project was also assisted by a grant from the Pennsylvania Department of Health using Tobacco Settlement Funds. Any opinions, findings, and conclusions or recommendations expressed in this material are those of the authors and do not necessarily reflect the views of the National Science Foundation or the National Institutes of Health.

\section{Author details}

${ }^{1}$ Center for Infectious Disease Dynamics and Department of Entomology, Merkle Lab, Pennsylvania State University, Orchard Road, University Park, PA 16802, USA. ${ }^{2}$ College of Veterinary Medicine, Odum School of Ecology, University of Georgia, DW Brooks Drive, Athens, GA 30602, USA.

Received: 24 July 2014 Accepted: 6 December 2014

Published online: 14 December 2014

\section{References}

1. World Malaria Report 2012. World Health Organization.

2. Garrett-Jones C: Prognosis for interruption of malaria transmission through assessment of the mosquito's vectorial capacity. Nature 1964, 204:1173-1175.

3. Smith DL, McKenzie FE: Statics and dynamics of malaria infection in Anopheles mosquitoes. Malar J 2004, 3:13.

4. Paaijmans KP, Blanford S, Chan BHK, Thomas MB: Warmer temperatures reduce the vectorial capacity of malaria mosquitoes. Biol Lett 2012, 8:465-468.

5. Mordecai EA, Paaijmans KP, Johnson LR, Balzer C, Ben-Horin T, de Moor E, McNally A, Pawar S, Ryan SJ, Smith TC, Lafferty KD: Optimal temperature for malaria transmission is dramatically lower than previously predicted. Ecol Lett 2013, 16:22-30.

6. Afrane YA, Little TJ, Lawson BW, Githeko AK, Yan G: Deforestation and vectorial capacity of Anopheles gambiae Giles mosquitoes in malaria transmission, Kenya. Emerg Infect Dis 2008, 14:1533-1538.

7. Afrane YA, Githeko AK, Yan G: The ecology of Anopheles mosquitoes under climate change: case studies from the effects of deforestation in East African highlands. Ann N Y Acad Sci 2012, 1249:204-210.

8. Fontenille D, Simard F: Unravelling complexities in human malaria transmission dynamics in Africa through a comprehensive knowledge of vector populations. Comp Immunol Microbiol Infect Dis 2004, 27:357-375.

9. Paaijmans KP, Read AF, Thomas MB: Understanding the link between malaria risk and climate. Proc Natl Acad Sci U S A 2009, 106:13844-13849.

10. Paaijmans KP, Blanford S, Bell AS, Blanford JI, Read AF, Thomas MB: Influence of climate on malaria transmission depends on daily temperature variation. Proc Natl Acad Sci U S A 2010, 107:15135-15139.

11. Blanford JI, Blanford S, Crane RG, Mann ME, Paaijmans KP, Schreiber KV, Thomas MB: Implications of temperature variation for malaria parasite development across Africa. Sci Rep 2013, 3:1300.

12. Afrane YA, Lawson BW, Githeko AK: Effects of microclimatic changes caused by land use and land cover on duration of gonotrophic cycles of Anopheles gambiae (Diptera: Culicidae) in Western Kenya Highlands. J Med Entomol 2005, 42:974-980

13. Parham PE, Michael E: Modeling the effects of weather and climate change on malaria transmission. Environ Health Perspect 2010, 118:620-626.

14. Okech BA, Gouagna LC, Yan G, Githure Jl, Beier JC: Larval habitats of Anopheles gambiae s.s. (Diptera: Culicidae) influences vector competence to Plasmodium falciparum parasites. Malar J 2007, 6:50.

15. Lyimo EO, Takken W, Koella JC: Effect of rearing temperature and larval density on larval survival, age at pupation and adult size of Anopheles gambiae. Entomol Exp Appl 1992, 63:265-271.

16. Zakharova NF, Losev Gl, lakubovich VI: The effect of density and temperature on the larval populations of the malaria vector Anopheles sacharovi. Med Parazitol (Mosk) 1990, 1:3-7.

17. Dye $C$ : The analysis of parasite transmission by bloodsucking insects. Annu Rev Entomol 1992, 37:1-19.

18. Dye C: The epidemiological context of vector control. Trans $R$ Soc Trop Med Hyg 1994, 88:147-149.

19. Suwanchaichinda C, Paskewitz SM: Effects of larval nutrition, adult body size, and adult temperature on the ability of Anopheles gambiae (Diptera: Culicidae) to melanize Sephadex beads. J Med Entomol 1998, 35(2):157-161

20. Aboagye-Antwi F, Tripet F: Effects of larval growth condition and water availability on desiccation resistance and its physiological basis in adult Anopheles gambiae sensu stricto. Malar J 2010, 9:225.

21. Afrane YA, Zhou G, Lawson BW, Githeko AK, Yan G: Life-table analysis of Anopheles arabiensis in western Kenya highlands: effects of land covers on larval and adult survivorship. Am J Trop Med Hyg 2007, 77:660-666. 
22. Ellers J, Jervis M: Body size and the timing of egg production in parasitoid wasps. Oikos 2003, 102:164-172.

23. Pechenik JA, Wendt DE, Jarrett JN: Metamorphosis Is not a new beginning: larval experience influences juvenile performance. Bioscience 2011, 48:901-910.

24. De Block M, Stoks R: Short-term larval food stress and associated compensatory growth reduce adult immune function in a damselfly. Ecol Entomol 2008, 33:796-801.

25. Schulte-Hostedde Al, Zinner B, Millar JS, Graham J: Restitution of mass-size residuals: validating body condition indices. Ecology 2013, 86:155-163.

26. Kingsolver JG, Huey RB: Size, temperature, and fitness: three rules. Evol Ecol Res 2008, 10:251-268.

27. Honek A: Intraspecific variation in body size and fecundity in insects: a general relationship. Oikos 1993, 66:483-492.

28. Kingsolver JG, Pfennig DW: Individual-level selection as a cause of Cope's rule of phyletic size increase. Evolution (N Y) 2004, 58:1608-1612.

29. Plaistow SJ, Troussard JP, Cézilly F: The effect of the acanthocephalan parasite Pomphorhynchus laevis on the lipid and glycogen content of its intermediate host Gammarus pulex. Int J Parasitol 2001, 31:346-351.

30. Pulkkinen K, Ebert D: Host starvation decreases parasite load and mean host size in experimental populations. Ecology 2004, 85:823-833.

31. Moret $Y$, Schmid-Hempel P: Survival for immunity: the price of immune system activation for bumblebee workers. Science 2000, 290(80):1166-1168,

32. Briegel $\mathrm{H}$ : Metabolic relationship between female body size, reserves, and fecundity of Aedes aegypti. J Insect Physiol 1990, 36:165-172.

33. Zhou G, Pennington JE, Wells MA: Utilization of pre-existing energy stores of female Aedes aegypti mosquitoes during the first gonotrophic cycle. Insect Biochem Mol Biol 2004, 34:919-925.

34. Levi T, Ben-Dov E, Shahi P, Borovsky D, Zaritsky A: Growth and development of Aedes aegypti larvae at limiting food concentrations. Acta Trop 2014, 133:42-44.

35. Alto BW, Lounibos LP, Mores CN, Reiskind MH: Larval competition alters susceptibility of adult Aedes mosquitoes to dengue infection. Proc Biol Sci 2008, 275:463-471.

36. Alto BW, Lounibos LP, Higgs S, Juliano SA: Larval competition differentially affects arbovirus infection in Aedes mosquitoes. Ecology 2005, 86:3279-3288.

37. Telang A, Qayum AA, Parker A, Sacchetta BR, Byrnes GR: Larval nutritional stress affects vector immune traits in adult yellow fever mosquito Aedes aegypti (Stegomyia aegypti). Med Vet Entomol 2012, 26:271-281.

38. Paaijmans KP, Huijben S, Githeko AK, Takken W: Competitive interactions between larvae of the malaria mosquitoes Anopheles arabiensis and Anopheles gambiae under semi-field conditions in western Kenya. Acta Trop 2009, 109:124-130.

39. Takken W, Smallegange RC, Vigneau AJ, Johnston V, Brown M, Mordue-Luntz A, Billingsley PF: Larval nutrition differentially affects adult fitness and Plasmodium development in the malaria vectors Anopheles gambiae and Anopheles stephensi. Parasit Vectors 2013, 6:345.

40. Araújo M, Gil LH, E-Silva A: Larval food quantity affects development time, survival and adult biological traits that influence the vectorial capacity of Anopheles darlingi under laboratory conditions. Malar J 2012, 11:261.

41. Nasci R: Relationship of wing length to adult dry weight in several mosquito species (Diptera: Culicidae). J Med Entomol 1990, 27:716-719.

42. Lyimo EO, Takken W: Effects of adult body size on fecundity and the pregravid rate of Anopheles gambiae females in Tanzania. Med Vet Entomol 1993, 7:328-332

43. Reisen WK, Aslamkhan M: A release-recapture experiment with the malaria vector, Anopheles stephensi Liston, with observations on dispersal, survivorship, population size, gonotrophic rhythm and mating behavior. Ann Trop Med Parasitol 1979, 73(3):251-69.

44. Detinova T: Age structure of Insect Populations of Medical Importance. Annu Rev Entomol 1966, 13:247

45. Clements A, Paterson GD: The analysis of mortality and survival rates in wild populations of mosquitoes. J Appl Ecol 1981, 18:373-399.

46. Smith DL, Battle KE, Hay SI, Barker CM, Scott TW, McKenzie FE: Ross, macdonald, and a theory for the dynamics and control of mosquitotransmitted pathogens. PLoS Pathog 2012, 8:e1002588.

47. Bell AS, Blanford S, Jenkins N, Thomas MB, Read AF: Real-time quantitative PCR for analysis of candidate fungal biopesticides against malaria: technique validation and first applications. J Invertebr Pathol 2009, 100:160-168.

48. Hard JJ, Bradshaw WE, Malarkey DJ: Resource- and density-dependent development in tree-hole mosquitoes. Oikos 1989, 54:137-144.
49. Grieco JP, Rejmánková E, Achee NL, Klein CN, Andre R, Roberts D: Habitat suitability for three species of Anopheles mosquitoes: Larval growth and survival in reciprocal placement experiments. J Vector Ecol 2007, 32:176-187.

50. Russell TL, Lwetoijera DW, Knols BGJ, Takken W, Killeen GF, Ferguson HM: Linking individual phenotype to density-dependent population growth: the influence of body size on the population dynamics of malaria vectors. Proc Biol Sci 2011, 278:3142-3151.

51. Hawley W: The effect of larval density on adult longevity of a mosquito, Aedes sierrensis: epidemiological consequences. J Anim Ecol 2010, 54:955-964.

52. Ameneshewa B, Service M: The relationship between female body size and survival rate of the malaria vector Anopheles arabiensis in Ethiopia. Med Vet Entomol 1996, 10:170-172.

53. Armbruster $P$, Hutchinson RA: Pupal mass and wing length as indicators of fecundity in Aedes albopictus and Aedes geniculatus (Diptera: Culicidae). J Med Entomol 2002, 39:699-704.

54. Andersson $\mathrm{HI}$ : The effect of sugar meals and body size on fecundity and longevity of female Aedes communis(Diptera: Culicidae). Physiol Entomol 1992, 17:203-207.

55. Gimnig JE, Ombok M, Otieno S, Kaufman MG, Vulule JM, Walker ED: Densitydependent development of Anopheles gambiae (Diptera: Culicidae) larvae in artificial habitats. J Med Entomol 2002, 39:162-172.

56. Ng'habi KR, John B, Nkwengulila G, Knols BGJ, Killeen GF, Ferguson HM: Effect of larval crowding on mating competitiveness of Anopheles gambiae mosquitoes. Malar J 2005, 4:49.

57. Muriu SM, Coulson T, Mbogo CM, Godfray HCJ: Larval density dependence in Anopheles gambiae s.s., the major African vector of malaria. J Anim Ecol 2013, 82:166-174.

58. Dye C: Vectorial capacity: must we measure all its components? Parasitol Today 1986, 2:203-209.

59. Dye C, Hasibeder G: Population dynamics of mosquito-borne disease: effects of flies which bite some people more frequently than others. Trans R Soc Trop Med Hyg 1986, 80:69-77.

60. Hancock RG, Foster WA: Larval and adult nutrition effects on blood/ nectar choice of Culex nigripalpus mosquitoes. Med Vet Entomol 1997 11:112-122.

61. Yaro AS, Touré AM, Guindo A, Coulibaly MB, Dao A, Diallo M, Traoré SF: Reproductive success in Anopheles arabiensis and the $M$ and $S$ molecular forms of Anopheles gambiae: do natural sporozoite infection and body size matter? Acta Trop 2012, 122:87-93.

62. Fernandes L, Briegel H: Reproductive physiology of Anopheles gambiae and Anopheles atroparvus. J Vector Ecol 2005, 30:11-26.

63. Hogg JC, Hurd H: Malaria-induced reduction of fecundity during the first gonotrophic cycle of Anopheles stephensi mosquitoes. Med Vet Entomol 1995, 9:176-180

64. Yuval B: Effect of body size on swarming behavior and mating success of male anopheles freeborni. J Insect Behav 1993, 6:333-342.

65. Yuval B: Mating systems of blood-feeding flies. Annu Rev Entomol 2006 51:413-440

66. Okanda FM, Dao A, Njiru BN, Arija J, Akelo HA, Touré Y, Odulaja A Beier JC, Githure JI, Yan G, Gouagna LC, Knols BGJ, Killeen GF: Behavioural determinants of gene flow in malaria vector populations: Anopheles gambiae males select large females as mates. Malar J 2002, 1:10.

67. Ponlawat A, Harrington LC: Age and body size influence male sperm capacity of the dengue vector Aedes aegypti (Diptera: Culicidae). J Med Entomol 2007, 44:422-426.

68. Stoffolano JG, Gonzalez EY, Sanchez M, Kane J, Oquendo AL, Sakolsky G, Schafer P, Yin C, Vela K: Relationship between size and mating success in the blow fly Phormia regina (Diptera: Calliphoridae). Ann Entomol Soc Am 2000, 93:673-677.

69. Vaughan JA: Population dynamics of Plasmodium sporogony. Trends Parasitol 2007, 23:63-70

70. Billingsley PF, Medley GF, Charlwood D, Sinden RE: Relationship between prevalence and intensity of Plasmodium falciparum infection in natural populations of Anopheles mosquitoes. Am J Trop Med Hyg 1994, 51:260-270.

71. Dong Y, Aguilar R, Xi Z, Warr E, Mongin E, Dimopoulos G: Anopheles gambiae immune responses to human and rodent Plasmodium parasite species. PLoS Pathog 2006, 2:e52.

72. Garver LS, Dong Y, Dimopoulos G: Caspar controls resistance to Plasmodium falciparum in diverse anopheline species. PLOS Pathog 2009, 5:e1000335. 
73. Joy DA, Gonzalez-Ceron L, Carlton JM, Gueye A, Fay M, McCutchan TF, Su X: Local adaptation and vector-mediated population structure in Plasmodium vivax malaria. Mol Biol Evol 2008, 25:1245-1252.

74. Caragata EP, Rancès E, Hedges LM, Gofton AW, Johnson KN, O'Neill SL, McGraw EA: Dietary cholesterol modulates pathogen blocking by Wolbachia. PLoS Pathog 2013, 9:e1003459.

75. Rivero A, Ferguson HM: The energetic budget of Anopheles stephensi infected with Plasmodium chabaudi: is energy depletion a mechanism for virulence? Proc Biol Sci 2003, 270:1365-1371.

76. Preuss J, Jortzik E, Becker K: Glucose-6-phosphate metabolism in Plasmodium falciparum. IUBMB Life 2012, 64:603-611.

77. Rono MK, Whitten MMA, Oulad-Abdelghani M, Levashina EA, Marois E: The major yolk protein vitellogenin interferes with the anti-plasmodium response in the malaria mosquito Anopheles gambiae. PLOS Biol 2010, 8:e1000434.

78. Takken W, Klowden MJ, Chambers GM: Effect of body size on host seeking and blood meal utilization in Anopheles gambiae sensu stricto (Diptera: Culicidae): the disadvantage of being small. J Med Entomol 1998, 35:639-645.

79. Ng'habi KR, Huho BJ, Nkwengulila G, Killeen GF, Knols BGJ, Ferguson HM: Sexual selection in mosquito swarms: may the best man lose? Anim Behav 2008, 76:105-112.

80. Kollien AH, Schaub GA: The development of Trypanosoma cruzi in triatominae. Parasitol Today 2000, 16:381-387.

81. Krist AC, Jokela J, Wiehn J, Lively CM: Effects of host condition on susceptibility to infection, parasite developmental rate, and parasite transmission in a snail-trematode interaction. J Evol Biol 2003, 17:33-40.

82. Logan A, Ruiz-González MX, Brown MJF: The impact of host starvation on parasite development and population dynamics in an intestinal trypanosome parasite of bumble bees. Parasitology 2005, 130:637-642.

83. Garrett-Jones C, Grab B: The Assessment of Insecticidal Impact on the Malaria Mosquito 's Vectorial Capacity, from Data on the Proportion of Parous Females. Bull World Health Organ 1964, 31:71-86.

84. Molineaux L, Shidrawi GR, Clarke J, Boulzaguet J, Ashkar T: Assessment of insecticidal impact on the malaria mosquito's vectorial capacity, from data on the man-biting rate and age-composition. Bull World Health Organ 1979, 57:265-274.

85. Bayoh MN, Mathias DK, Odiere MR, Mutuku FM, Kamau L, Gimnig JE, Vulule JM, Hawley WA, Hamel MJ, Walker ED: Anopheles gambiae: historical population decline associated with regional distribution of insecticidetreated bed nets in western Nyanza Province, Kenya. Malar J 2010, 9:62.

86. Chitnis N, Schapira A, Smith T, Steketee R: Comparing the effectiveness of malaria vector-control interventions through a mathematical model. Am J Trop Med Hyg 2010, 83:230-240.

87. ljumba JN, Lindsay SW: Impact of irrigation on malaria in Africa: paddies paradox. Med Vet Entomol 2001, 15:1-11.

88. Ferguson HM, Dornhaus A, Beeche A, Borgemeister C, Gottlieb M, Mulla MS, Gimnig JE, Fish D, Killeen GF: Ecology: a prerequisite for malaria elimination and eradication. PLoS Med 2010, 7:e1000303.

doi:10.1186/s13071-014-0593-4

Cite this article as: Moller-Jacobs et al: Capacity of mosquitoes to transmit malaria depends on larval environment. Parasites \& Vectors 2014 7:593.

\section{Submit your next manuscript to BioMed Central and take full advantage of:}

- Convenient online submission

- Thorough peer review

- No space constraints or color figure charges

- Immediate publication on acceptance

- Inclusion in PubMed, CAS, Scopus and Google Scholar

- Research which is freely available for redistribution 